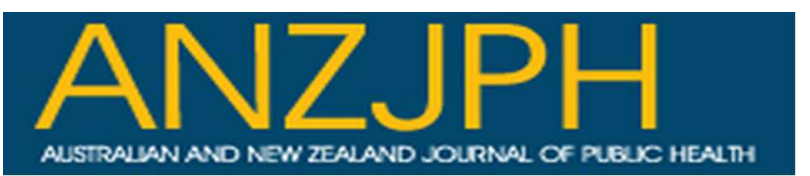

\title{
Establishing a process for conducting cross-jurisdictional record linkage in Australia
}

\begin{tabular}{|r|l|}
\hline Journal: & Australian \& New Zealand Journal of Public Health \\
\hline Manuscript ID: & ANZJPH-2015-114.R1 \\
\hline Wiley - Manuscript type: & Original Article \\
\hline Key words: & data linkage, immunisation, cross-jurisdiction \\
\hline & $\begin{array}{l}\text { Objective: To describe the realities of conducting a cross-jurisdictional data } \\
\text { linkage project involving state and Australian Government-based data } \\
\text { collections in order to inform future national data linkage programs of } \\
\text { work. } \\
\text { Methods: We outline the processes involved in conducting a Proof of } \\
\text { Concept data linkage project including the implementation of national data } \\
\text { integration principles, data custodian and ethical approval requirements, } \\
\text { and establishment of data flows. } \\
\text { Results: The approval process involved 9 approval and regulatory bodies } \\
\text { and took over } 2 \text { years. Data will be linked across 12 datasets involving 3 } \\
\text { data linkage centres. A framework was established to allow data to flow } \\
\text { between these centres while maintaining the separation principle which } \\
\text { serves to protect the privacy of the individual. } \\
\text { Conclusions: This will be the first project to link child immunisation records } \\
\text { from an Australian Government dataset to other administrative health } \\
\text { datasets for a population cohort covering } 2 \text { million births in } 2 \text { Australian } \\
\text { states. } \\
\text { Implications: Although the project experienced some delays, positive } \\
\text { outcomes were realised, primarily the development of strong collaborations } \\
\text { across key stakeholder groups including community engagement. We have } \\
\text { identified several recommendations and enhancements to this now } \\
\text { established framework to further streamline the process for data linkage } \\
\text { studies involving Australian Government data. }\end{array}$ \\
\hline
\end{tabular}




\begin{abstract}
Objective: To describe the realities of conducting a cross-jurisdictional data linkage project involving state and Australian Government-based data collections in order to inform future national data linkage programs of work.
\end{abstract}

Methods: We outline the processes involved in conducting a Proof of Concept data linkage project including the implementation of national data integration principles, data custodian and ethical approval requirements, and establishment of data flows.

Results: The approval process involved 9 approval and regulatory bodies and took over 2 years. Data will be linked across 12 datasets involving 3 data linkage centres. A framework was established to allow data to flow between these centres while maintaining the separation principle which serves to protect the privacy of the individual.

Conclusions: This will be the first project to link child immunisation records from an Australian Government dataset to other administrative health datasets for a population cohort covering 2 million births in 2 Australian states.

Implications: Although the project experienced some delays, positive outcomes were realised, primarily the development of strong collaborations across key stakeholder groups including community engagement. We have identified several recommendations and enhancements to this now established framework to further streamline the process for data linkage studies involving Australian Government data. 


\section{Introduction}

\section{Linkage of Administrative Health Datasets in Australia}

In order to obtain the most accurate, reliable, unbiased information to guide public health policy and practice, data on the total population collated from various data sources are needed. ${ }^{1}$ Data linkage is the process whereby records that are derived from different sources but relate to the same individual are linked together using a best-practice protocol, which ensures that individual privacy is rigorously protected. ${ }^{2}$ Data are usually derived from large population-based administrative health datasets containing information on a series of health services and outcomes. Linkage of these datasets is cost and time effective compared with conducting prospective cohort studies and can provide a rich and representative picture of the population. Data linkage projects can therefore contribute to substantial health improvements through informing the development of policy, and evaluation of services and improvements to clinical practice. ${ }^{3}$

Globally, there are few countries who have with the capabilities of conductinged large-scale data linkage projects. Australia is one such country, with established state and national data linkage units, including the Western Australian Data Linkage System, WADLS, ${ }^{4,5}$ the Centre for Health Record Linkage, CHeReL, ${ }^{6}$ in New South Wales (NSW) and the Australian Institute of Health and Welfare (AIHW). ${ }^{7}$ Although data linkage has been ongoing in WA since the 1970s, the WADLS was established in 1995 to centralise the development of linkage infrastructure. Since then more than $_{2}>900$ data linkage projects have used this facility. There are more than $>50$ million links in the WADLS, joining administrative, clinical and service data including all birth and death registrations, hospital inpatient, emergency, midwives and mental health records, notifiable infectious diseases, cancer registry, birth 
defects registry as well as a suite of non-health datasets including electoral roll, education, housing, child protection, corrective services and transport data. ${ }^{8}$

The CHeReL in NSW was established in 2006 and modelled on the processes developed for the WADLS. There are currently more than $>94$ million records across 17 core datasets including birth, death, perinatal, hospital inpatient, emergency, notifiable conditions, cancer registrations and mental health ambulatory care data in NSW and the Australian Capital Territory. ${ }^{6}$ The AIHW has undertaken linkage since the 1990 s, and has predominately focused on linkage with the National Death Index and the Australian Cancer Database.

Most administrative health datasets fall under the jurisdiction of state governments while some, such as Medicare-related data, which includes immunisation records from the Australian Childhood Immunisation Register (ACIR) and prescribing data from the Pharmaceutical Benefits Scheme (PBS), fall under the jurisdiction of the Australian Government. In order for state and Commonwealth data collections to be brought together, links need to be established using sensitive personal information such as name, address and date of birth. The Privacy Act (1988) ${ }^{9}$ balances the public interest in research, or public good, with the public interest in privacy. Additionally, the Freedom of Information Act in Australia, formed in 1982 and reformed in 2010, safeguards the right to access government information as well as the right to individuals' privacy. ${ }^{10}$ The intersection of these two basic rights is өften where pPopulation-based data linkage for public health research takes both these basic

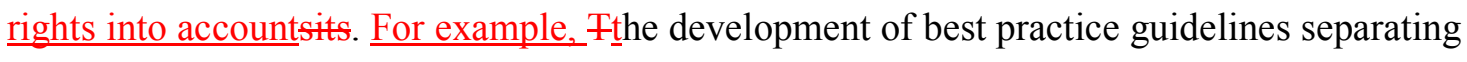
sensitive datapersonal information (identifying details such as name and address) from health service, or content data, in a framework that allows researchers to access only content data and linkage units to access only the personal informationsensitive data required for creating 
the links between records in separate datasets, ${ }^{2}$ has aided in overcoming the threats to individuals' privacy. However, until recently there has been no systematic process for researchers to be given approval to access data held in data collections managed by the Australian Government using best-practice guidelines. ${ }^{11}$

The Population Health Research Network (PHRN) is an initiative established by the Australian Government as part of the National Collaborative Research Infrastructure Strategy to facilitate data linkage activities across Australia. ${ }^{12}$ One such activity of the PHRN was the formation of Proof of Concept (POC) Collaborations to formally test multi-jurisdictional data linkage capabilities. POC1 and POC2, involved linkage of datasets across Australian states and have been described previously. ${ }^{13}$ The premise behind POC4 was cross-jurisdictional linkage of datasets between two or more Australian states and one or more Commonwealth datasets in order to investigate an issue of public health importance. We developed a program of work around evaluating Australia's National Immunisation Program through the linkage of birth, perinatal, death, hospital, emergency department, disease notification, routine laboratory and immunisation records (from ACIR) for a cohort of approximately 2 million births in NSW and WA. This will represent the first time cross-jurisdictional linkages have occurred between datasets from multiple Australian states and an Australian Government dataset under new Commonwealth arrangements for statistical data integration. ${ }^{14}$

The legal ramifications of data linkage involving Australian Government datasets has been discussed previously. ${ }^{15}$ In this article, we present the framework that was established during POC4 for conducting a cross-jurisdictional data linkage project in Australia involving an Australian Government dataset following the new Commonwealth arrangements. The framework includes establishing processes for data custodian and ethical approval and for 
data exchange between linkage centres. We discuss our community consultation process, where confirmed community support is a necessary pre-requisite for Aboriginal Health Ethics approval and a recommended pre-requisite in other National Health and Medical Research Council funded studies, even where 'community' might refer to the whole population. Finally, we offer some reflections on our experience and recommendations to further streamline the process which should be helpful for other research groups embarking on similar studies. Synonymous terms with data linkage are 'record linkage' and 'data integration'. Throughout this article we use the terms 'linkage' and 'integration' interchangeably as their use varies between centres.

\section{Methods}

The key data sources for our project were WA and NSW birth registrations (to establish the cohort of children), the ACIR (providing the immunisation status of each child) and routinely collected health outcome datasets in WA and NSW (e.g. hospitalisation, emergency department presentations and infectious disease notifications). Several processes were required in order to obtain custodian release of ACIR data from the Australian Government and then establish linkages between all sources of data.

\section{Commonwealth Arrangements for Statistical Data Integration involving}

\section{Commonwealth Data}

In 2010, the heads of all Australian Government agencies and the Australian Public Service Commission endorsed a set of high level principles to govern the linkage of Commonwealth data for statistical and research purposes. ${ }^{14}$ This also included a set of governance and institutional arrangements to support these principles. The seven high level principles cover treating administrative data as a strategic resource, the accountability of data custodians, data 
integrators and investigators, the public benefit and purposes of the proposed linkage activity, the preservation of privacy and confidentiality, and public transparency. ${ }^{16}$ The first of these principles notes that the statistical and research value of administrative data, as a strategic resource, should be maximised by granting broad access for research purposes to data that is not likely to enable re-identification. Another key requirement is the need for projects using data from an Australian Government collection deemed to be 'high risk' to be linked through an accredited Integrating Authority. ${ }^{14}$ At the time of establishing our project, there were two accredited Commonwealth Integrating Authorities: the AIHW (accredited on 28 June 2012) and the Australian Bureau of Statistics (accredited on 24 April 2012). For our project, the AIHW was appointed as the Integrating Authority by the Department of Health.

\section{Data Custodian and Ethical Approval Process}

In order to conduct this project involving data from WA, NSW and the Australian Government, several data linkage centres were involved. These were the established data linkage centres in WA (WADLS) and NSW (CHeReL) and the approved Integrating Authority, the AIHW. Approvals were required from each of these linkage centres, their associated data custodians and Human Research Ethics Committees (HREC) from the departments of health in each jurisdiction. Some of these bodies accepted completion of the National Ethics Application Form (NEAF) while others requested completion of their own application forms. Due to the disproportionate burden of vaccine-preventable disease in Aboriginal children, our research team proposed to examine vaccine coverage, timeliness and effectiveness in Aboriginal and non-Aboriginal children separately. Therefore, specific Aboriginal HREC approvals were also required in WA and NSW. All approvals required for the project are listed in Table 1. 


\section{Public Interest Certificate}

Obtaining a Public Interest Certificate, or PIC, was a pivotal step in our project. The PIC serves as the data custodian approval for release of data for linkage that is governed by the Australian Government, such as the ACIR. Initially it was unclear whose responsibility it was to initiate this process, whether it was the research team requesting access to the data, the Integrating Authority, or the ACIR data custodian. Aside from one previously signed PIC associated with investigating vaccine safety also using ACIR data, ${ }^{17}$ there was no clear process for requesting a PIC or what information a PIC request should contain. Over a three year period, a template was developed and the roles and responsibilities for obtaining a PIC were clarified. This was achieved through a collaborative effort between the Department of Health (who facilitates sign off by the custodian), Department of Human Services (who manage the ACIR), the AIHW, PHRN and the research team. The final version of the PIC, signed by the Chief Medical Officer pursuant to the National Health Act 1953 and the Health Insurance Act 1973, under which ACIR is administered, certifies that it is in the public interest for a) identifying details from ACIR to delivered to the AIHW for linkage purposes, b) content data from ACIR to be delivered to AIHW for merging purposes and c) the linked content data to be delivered to the research team.

\section{Secure Data Laboratory and Integration Checks}

A further requirement to use data governed by the Australian Government is for Integrating Authorities to ensure sound data management and secure data access to researchers. One of

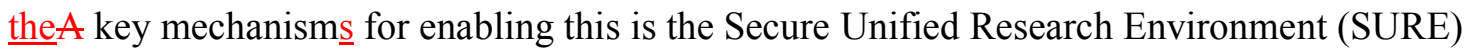
system, a remote data access laboratory, operated by the Sax Institute in Sydney, NSW. This allows researchers to access and analyse data through a remote access computing environment with comprehensive security features and a curated gateway to inspect data files 
entering and leaving the secure workspace. ${ }^{18} \mathrm{~A}$ further privacy feature is the inability for individuals to access the internet, printers or removable media while they are logged into the facility. Before individual researchers can apply for access to establish a SURE workspace, they must complete a training program to become accredited SURE users.

Integrating Authorities are obligated to conduct data integration checks of the linkable datasets prior to release to researchers to ensure the data, when other protective measures such as use of SURE are considered, are not likely to re-identify individuals. One possibility to allow these data integration checks was for all content data to be sent directly to AIHW for checking before being released to the SURE system. However, this was deemed as not appropriate nor practical by all the parties involved. The agreed solution was that content data would be transferred onto SURE by either the respective linkage unit or the data custodian and then data integration checks would be performed by AIHW staff within the SURE workspace prior to the research team having access to the data (Figure 1). This agreed process minimised the transfer of content data between linkage units but enabled AIHW to conduct data integration checks prior to release of the content data to researchers.

\section{Community Engagement}

Community engagement is integral to the successful and ethical conduct of projectsresearch, including projects involving population-based linked data. Our research team consulted with two established community groups in WA. Firstly, we consulted with the Infectious Diseases Community Reference Group, established in 2007 at the Telethon Kids Institute. This group has the mission of informing the wider community of current and proposed research projects involving infectious diseases and vaccination, aid in dissemination of research outcomes and for community members to provide valuable input in all stages of the research process. 
Membership of this group includes 8-10 Aboriginal and non-Aboriginal community members with extensive links to other Aboriginal and non-Aboriginal community organisations throughout WA. Secondly, we consulted with the Aboriginal Collaborative Council Advising Research and Evaluation, which has the primary goal of facilitation, translating and applying Aboriginal research findings into policy and practice in WA. The proposed study including all ethical requirements and details regarding access to sensitive data was discussed. Both these groups saw no threat to privacy or other issues with the project and subsequently provided letters of support. Unlike WA, there were no pre-existing community reference groups in NSW. Therefore, the research team set out to establish a national Aboriginal Immunisation Reference Group with membership including individuals from Aboriginal medical services, Aboriginal health worker organisations, Aboriginal Health Councils and the National Centre for Immunisation Research and Surveillance. Community consultation with these groups will continue throughout the life of the project.

\section{Results}

\section{Timeline of Events}

The length of time between formal submission and approval from the different regulatory bodies is illustrated in Table 1. From the time of submission of the first application to approval of the final application was approximately 2 years. While some regulatory bodies granted approval in a matter of weeks, others took several months. As a result of the length of time between submission and approval, annual reports for some ethics committees were due prior to all approvals being granted from the linkage units, that is, one full year had lapsed between approval of an ethics committee and approval of another regulatory body. Additionally, due to the length of time to obtain all approvals, several changes occurred within organisations which resulted in the need for ethical and data custodian approval for 
amendments to the project. For example, the NSW Ministry of Health conducted a review in early 2014 of the NSW Admitted Patient Data Collection with the purpose of streamlining access to data in NSW. The review altered the data variables that were approved for release to researchers. Additionally, a more definitive variable list became available for the NSW Birth Register data. While this will substantially benefit future linkage projects, approval from the relevant data custodians needed to be re-sought for our project, and an ethics amendment to the NSW Population \& Health Services Research Ethics Committee was required.

Another example of delays relates to the fact that the full data linkage application in WA could not be granted approval by the WA data custodians until the required processes for data flow including the process for the data integration checks were agreed upon from all parties. This required numerous negotiations between the data custodians in each jurisdiction. As a result of this length of time, the preliminary approval with the data custodians, in particular the WA Registry of Births, Deaths and Marriages (which forms the basis of the study cohort), had lapsed. Unlike the initial approval, a change in procedures meant that re-approval required a formal Memorandum of Understanding between the Registry of Births, Deaths and Marriages in WA and the AIHW, which further delayed the project. By August 2014, all necessary approvals and amendments had been completed and data extraction could take place.

\section{Establishment of Data Flows}

The key outcome of POC4 was the establishment of a process for data flows and data integration checks between the three linkage units and to develop an order of events that was agreeable to all parties involved. The transfer of identifying data for linkage and content data 
for analysis between these three linkage units is presented in Figure 1. The study cohort is live births in WA and NSW between 1 January 1996 and 31 December 2012, based on registrations from the Register of Births in WA and NSW. The WADLS and CHeReL used existing linkages between the birth registers and the respective state datasets (hospital admissions, perinatal records, emergency department data, infectious disease notifications and routine laboratory data (WA only)) to assign state project-specific linkage keys to each dataset as per their usual practices. The AIHW was responsible for linking birth records to immunisation records from the ACIR and the National Death Index. This required the secure transfer of identifying details from the WA and NSW Register of Births to the AIHW linkage domain. The AIHW linkage strategy applied a general framework that has been previously $\underline{\text { described }}^{19}$ with some extensions such as a frequency based agreement weight for names and approximate string comparators. Concurrently, the identifying details for children on ACIR were transferred to the AIHW linkage domain. This 3-way linkage resulted in the creation of national project-specific linkage keys which connects individuals from each state to their corresponding immunisation records. The AIHW then created a mapping file containing individual national project-specific linkage keys and their corresponding WA or NSW project-specific linkage key. The Department of Human Services then extracted the content data from ACIR and AIHW uploaded these to the SURE system.

In parallel, the WA and NSW project-specific linkage keys were provided to each of the data custodians in WA and NSW with their original record identifiers. Each data custodian in WA and NSW then extracted the approved content data to be uploaded to SURE. Separate agreements have been set in place between CHeReL, NSW Ministry of Health, WADLS and the Sax Institute to enable state specific content data with their state project-specific linkage keys to be uploaded to SURE. Within each linkage unit, the separation principle is 
maintained at all times by storing the personal data used for linkage and the content data on

$\underline{\text { separate network domains which are only accessible by physically separated teams of }}$ approved staff.

\section{SURE Workspace}

Access to the SURE system by the study researchers required a once off service agreement that had to be ratified by the lead investigator's institution, an application for a SURE workspace, and a researcher deed for the use of SURE virtual desktops signed by each researcher requiring access to the workspace. These applications allowed the creation of a SURE workspace and the registration of the researchers involved in the project who will be responsible for the data analysis. Before the researchers were granted access to the linked content data within SURE, specifically allocated staff who were not involved in the linkage at the AIHW conducted data integrity checks to ensure that the data content is as approved to manage risks of re-identification. By March 2015, content data from ACIR and all bar one of the state datasets had been uploaded to the study specific SURE workspace and released to researchers for analysis.

\section{Discussion and Recommendations}

Accessing and bringing together information stored on administrative databases for the purposes of public health research is critical in order to evaluate and inform public health policy and ultimately improve the health and wellbeing of the population. In this article, we have described the processes involved in conducting a cross-jurisdictional data linkage project involving data from state and Australian Government datasets to evaluate Australia's National Immunisation Program. While we experienced lengthy delays whilst processes were being established, there have been several positive outcomes in addition to the successful 
linkage of ACIR and state datasets, including considerable progress towards developing a streamlined framework for conducting future cross-jurisdictional data linkage studies.

First, the process has resulted in the development of strong collaborations and partnerships between the research team (in particular the principal investigators in NSW and WA), data custodians, data linkage centres in each jurisdiction, the PHRN and state and Commonwealth departments of health. Second, we have developed a clear framework for the flow of data between organisations whilst also maintaining the separation principle ${ }^{2}$ which serves to protect the individual's privacy. To achieve this, a mutual understanding was developed between all parties of their respective views of separation principles and protocols that guide these principles. Third, we have strengthened our engagement with community groups. Through regular consultation with the Infectious Diseases Community Reference Group in WA there has been greater awareness of the time taken to undertake data linkage projects, and ethical and legal ramifications of conducting these projects. The research team will continue to engage with the Infectious Diseases Community Reference Group and the National Aboriginal Immunisation Reference Group, specifically established for this project.

The importance of including Commonwealth datasets in data linkage studies, in particular health related datasets such as the PBS and ACIR to allow the conduct of pharmacovigilance (including vaccine safety) and epidemiological studies, is well recognised. Earlier work conducted in WA using PBS data linked to the WA Birth Defects Registry for a population cohort of pregnant women 2002-2005 found several associations between medicines and birth defects. ${ }^{20}$ However, since the implementation of the high level principles to govern the linkage of Australian Government data in 2010, no other large scale studies using linked PBS data have been published and there has only been one published study using linked ACIR 
data in South Australia. ${ }^{17}$ This POC Collaboration has led to the development of a framework for conducting future data linkage projects, which will hopefully lead to increased availability of timely health data, especially for projects involving Commonwealth datasets. Based on our experience with this process, we propose the following recommendations to enhance timely access to linked health data, some of which are currently being implemented:

(1) Collaboration across national and state-based linkage infrastructure in Australia. A more unified approach to national data linkage projects between state and Commonwealth departments would result in streamlined project approvals and data access requests, in particular for cross-jurisdictional linkage projects. This would enable data to be delivered in a cost-effective and timely manner so outcomes from research projects are more relevant and rapidly translatable. While the processes associated with data utilisation are $\underline{\text { ultimately the responsibility of the jurisdictions involved, this project demonstrates that }}$ Australian jurisdictions can work within a common framework to deliver cross jurisdictional linked data. The Cross Portfolio Data Integration Oversight Board, the body responsible for Commonwealth data integration arrangements, $\underline{\text { has conducted is eurrently }}$ eonducting an independent review focusing on the efficiency of the Commonwealth arrangements for statistical data integration involving Commonwealth data- and the $\underline{\text { results are currently being considered. }}$

(2) Standardised ethical application forms across multiple jurisdictions. While the majority of the approval bodies would accept the NEAF, a committee specific application form was needed for the AIHW and the WA Aboriginal Health Ethics Committee, and additional modules for the NEAF were required for the state departments of health human research ethics committees. We recommend the formation of a single application with 
sections relevant to data linkage projects that can be reviewed by multiple ethics bodies across various jurisdictions. This would considerably reduce the time involved in establishing multiple jurisdictional data linkage projects and minimise the administrative burden experienced by all parties. Further to this, we would advocate for a single ethical review for future cross-jurisdictional projects to avoid the administrative burden of multiple annual reports to all ethical bodies. This recommendation has also been made by the Privacy Commissioner of British Columbia, a jurisdiction with similar populationbased data linkage infrastructure for health policy research, with regards to data linkage research projects. $^{21}$

(3) Process for and approval of a Public Interest Certificate. The PIC is the data custodian sign off for the use of Commonwealth data housed in an Australian Government dataset and was a critical milestone for our project. In any data linkage study, the data custodian of the specified dataset has the final decision regarding release of the data. This approval is not always guaranteed even though there may be no explicit technological, or ethical impediment to release of the data for research purposes. ${ }^{11}$ However, after it has been established that there are no technological or ethical impediments to the release of data, approval on any legislative and policy grounds, should be expedited. POC4 has resulted in the development of a template, and a clear and transparent process for future PICs for data linkage projects, which should expedite the process of data custodian sign off. Additionally, the AIHW has developed guidelines for researchers to assist in the progress of applications for Commonwealth data.

(4) Standardised application forms and nomenclature across data linkage centres. The terms 'core datasets', ‘master linkage key’, 'project-specific linkage key’, 'mapping file' have 
slightly different meanings across the different data linkage centres in each jurisdiction, which at times cause confusion leading to differing interpretations and delays in the application and approval process. We recommend standard nomenclature and application forms to streamline the approval process for cross-jurisdictional linkage projects. The PHRN have developed a web-based standardised data linkage application form (accessible at https://oas.phrn.org.au) that should significantly aid this process for future projects.

(5) Comprehensive metadata on datasets with data available for linkage. While researchers have access to data dictionaries on the various state data collections, often provided by the state linkage units, it would beneficial to have adequate metadata on available Australian Government datasets. Ideally these metadata would include variable lists, coding of categorical variables and indications of quality of individual data items. Furthermore, some data items are not standardised across Australia. For example, the Socio-Economic Index for Areas (SEIFA) scores and the Accessibility and Remoteness Index of Australia (ARIA) scores calculated at small geographical areas (such as collection districts and statistical local areas) provide the most accurate measures of socio-economic status and remoteness across Australia. These data items, based on geocoded residential addresses (rather than the cruder measures based on postcode or larger geographic boundaries), are currently only derived and available in WA which limits the scope for national comparisons.

\section{Conclusion}

Australia is one of the few countries where established data linkage infrastructure provides the capacity for conducting large-scale studies utilising population-based data collected at the 
state and Commonwealth level. Whilst further enhancements are recommended, the POC4 Collaboration has resulted in the development of strong partnerships between key stakeholders and the establishment of a framework to assist researchers with future crossjurisdictional linkage projects. This should ultimately lead to more timely access to data to inform health policy and monitor vaccine and drug safety and effectiveness.

\section{References}

1. Stanley FJ. Record linkage: Public good vs invasion of privacy. 25th International Conference of Data Protection and Privacy Commissioners; 10 September 2003; Sydney, Australia_2003.

2. Kelman CW, Bass AJ, Holman CD. Research use of linked health data--a best practice protocol. Aust N Z J Public Health. 2002;26(3):251-5.

3. Brook EL, Rosman DL, Holman CDAJ. Public good through data linkage: measuring research outputs from the Western Australian Data Linkage System. Aust N Z J Public Health. 2008;32(1):19-23.

4. Holman CD, Bass AJ, Rosman DL, Smith MB, Semmens JB, Glasson EJ, et al. A decade of data linkage in Western Australia: strategic design, applications and benefits of the WA data linkage system. Aust Health Rev. 2008;32(4):766-77.

5. Holman CD, Bass AJ, Rouse IL, Hobbs MS. Population-based linkage of health records in Western Australia: development of a health services research linked database. Aust N Z J Public Health. 1999;23(5):453-9. 
6. Centre for Health Record Linkage [Internet] 2014 [cited 2 October 2014]. Available from: http://www.cherel.org.au/.

7. Australian Institute of Health and Welfare. Data linkage at the AIHW. Access. 2012 [cited April 2012:8-9]. Available from http://www.aihw.gov.au/data-linking/

8. Data Linkage Western Austrralia [Internet] 2014 [cited 2 October 2014]. Available from: http://www.datalinkage-wa.org.au/.

9. Privacy Act, (1988).

10. Freedom of Information Act, (1982).

11. Adams C, Allen J. Government databases and public health research: facilitating access in the public interest. J Law Med. 2014;21(4):957-72.

12. Population Health Research Network [nternet] 2014[cited 18 November 2014]. Available from: www.phrn.org.au.

13. Mitchell RJ, Cameron CM, Ramback MR. Data linkage for injury surveillance and research in Australia: perils, pitfalls and potential. Aust N Z J Public Health. 2014;38(3):27580.

14. A guide for data integration projects involving Commonwealth data for statistical and research purposes: Australian Government; 2014[cited 2 October 2014]. Available from: http://statistical-data-integration.govspace.gov.au/.

15. Xafis V, Thomson C, Braunack-Mayer AJ, Duszynski KM, Gold MS. Legal impediments to data linkage. J Law Med. 2011;19(2):300-15.

16. Australian Government Cross Portfolio Statistical Integration Committee (CPSIC). High level principles for Integrating Commonwealth Data for Statistical Research Purposes. Australian Government; 2010.

17. Gold M, Dugdale S, Woodman RJ, McCaul KA. Use of the Australian Childhood Immunisation Register for vaccine safety data linkage. Vaccine. 2010;28(26):4308-11. 
18. Secure Unified Research Environment [Internet] 2014 [cited 2 October 2014]. Available from: https://www.sure.org.au/.

19. Fellegi I, Sunter A. A Theory for Record Linkage. Journal of the American Statistical Association. 1969;64:1183-210.

20. Colvin L, Slack-Smith L, Stanley FJ, Bower C. Linking a pharmaceutical claims database with a birth defects registry to investigate birth defect rates of suspected teratogens. Pharmacoepidemiol Drug Saf. 2010;19(11):1137-50.

21. Denham E. A Prescription for Legislative Reform: Improving Privacy Protection in BC's Health Sector. Office of the Informaiton and Privacy Commissioner for British Columbia, 2014. 
Figure Legends

FIGURE 1: Approved data flows between WA, NSW and AIHW data linkage centres 
TABLE 1: Approvals required for Australian Childhood Immunisation Register cross-jurisdictional POC4 linkage project

\begin{tabular}{|c|c|c|c|c|}
\hline Regulatory Body & Details & Jurisdiction & Submission Date & Approval Date \\
\hline $\begin{array}{l}\text { Western Australian Data Linkage Branch } \\
\text { (WADLB) }\end{array}$ & $\begin{array}{l}\text { WADLB Expression of } \\
\text { Interest (Draft } \\
\text { Application) }\end{array}$ & WA & 28 Sept 2012 & 7 Nov 2012 \\
\hline WADLB & $\begin{array}{l}\text { WADLB Full } \\
\text { Application Form }\end{array}$ & WA & 12 Nov 2012 & $21 \mathrm{Jul} 2014$ \\
\hline Centre for Health Record Linkage (CHeReL) & $\begin{array}{l}\text { CHeReL Application for } \\
\text { Data Form }\end{array}$ & NSW & 28 Sept 2012 & 29 Oct 2012 \\
\hline $\begin{array}{l}\text { Department of Health WA Human Research } \\
\text { Ethics Committee (DoHWA HREC) }\end{array}$ & $\begin{array}{l}\text { NEAF plus WA Specific } \\
\text { Module }\end{array}$ & WA & 27 Nov 2012 & 4 Feb 2013 \\
\hline $\begin{array}{l}\text { NSW Population \& Health Services Research } \\
\text { Ethics Committee }\end{array}$ & $\begin{array}{l}\text { NSW specific NEAF } \\
\text { plus NSW Specific } \\
\text { Model }\end{array}$ & NSW & $\begin{array}{c}\text { 14 Mar } 2013 \\
\text { Amendment: } 25 \text { Jul } 2014\end{array}$ & $\begin{array}{c}\text { 9 May } 2013 \\
\text { Amendment: } 5 \text { Aug } 2014\end{array}$ \\
\hline $\begin{array}{l}\text { WA Aboriginal Health Ethics Committee } \\
\text { (WAAHEC) }\end{array}$ & $\begin{array}{l}\text { WAAHEC Application } \\
\text { Form }\end{array}$ & WA & 25 Jan 2013 & 15 Feb 2013 \\
\hline
\end{tabular}


Council Ethics Committee (AH\&MRC)

\begin{tabular}{llll}
\hline Australian Government Department of Health & NEAF & Commonwealth & 14 Jan 2013 \\
and Ageing Departmental Ethics Committee & & & \\
(Health) & & & \\
\hline Australian Institute of Health and Welfare & AIHW form plus AIHW & Commonwealth & 16 Oct 2013 \\
(AIHW) & Undertaking & & \\
\hline Department of Health & Public Interest Certificate & Commonwealth & 7 Aug 2013* 2012
\end{tabular}

WA, Western Australia; NSW, New South Wales; NEAF, National Ethics Application Form

*Initial enquiries to obtain the Public Interest Certificate commence in 2011. 


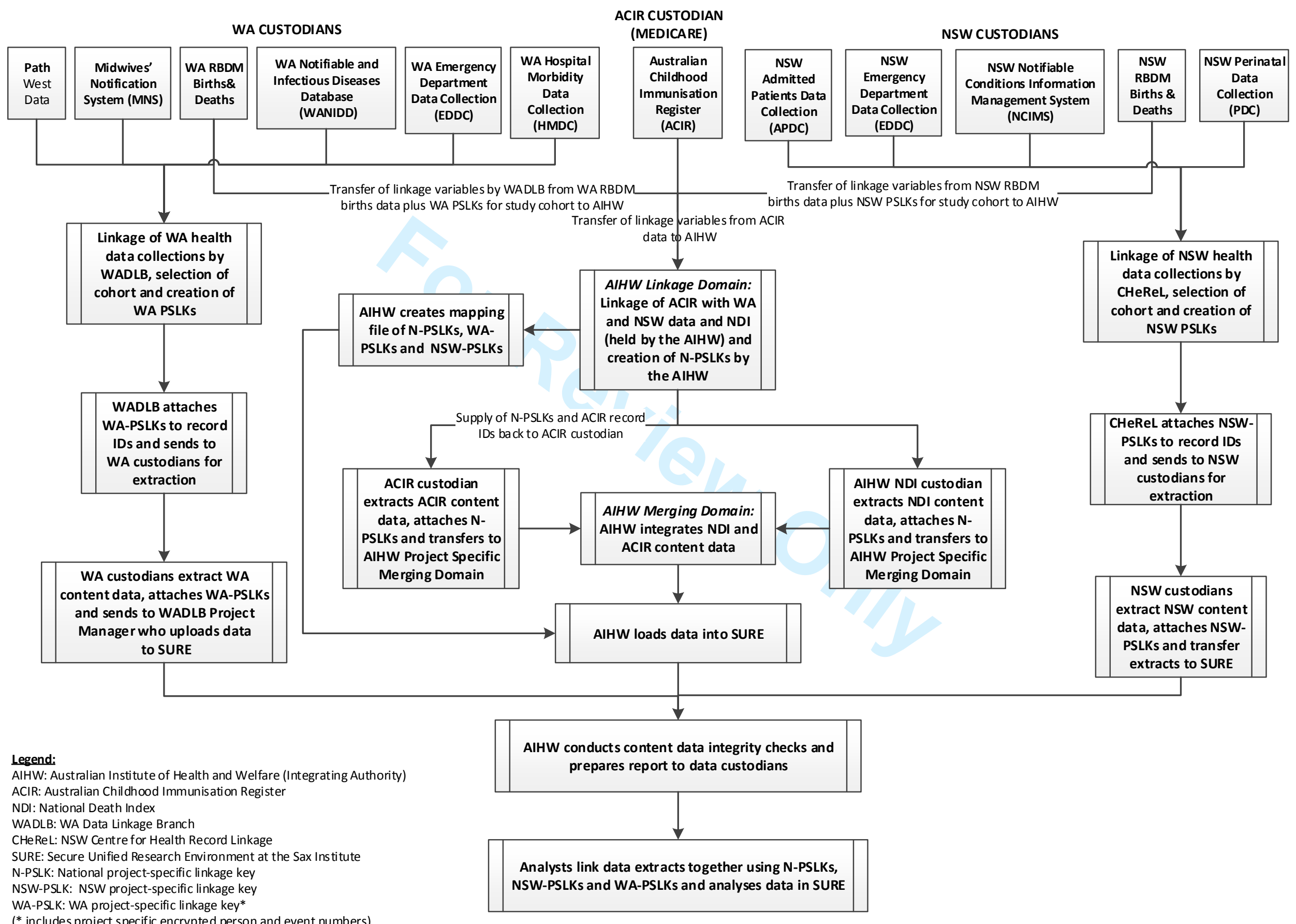

WA-PSLK: WA project-specific linkage key*

(* includes project specific encrypted person and event numbers) 